\title{
Treatment Effect of Donepezil is Greater in Moderate Stage Than in Mild Stage of Alzheimer's Disease
}

\author{
Min Soo Jung ${ }^{\mathrm{a}}$, Young Min Lee, b, c, Je Min Park ${ }^{\mathrm{a}}$, Byung Dae Lee \\ Eunsoo Moon ${ }^{\mathrm{a}}$, Young In Chung ${ }^{\mathrm{a}}$
}

\begin{abstract}
Background: Alzheimer's disease (AD) is a progressive disease with an insidious onset and a downhill course. Treatment response of cholinesterase inhibitors for AD seems to depend on severity degree but it is not clear which stage is more responsive.

Methods: This was a 1 year, retrospective cohort study. AD patients (very mild, 188; mild, 153; moderate, 31) were recruited from the memory impairment clinic at Pusan National University Hospital of South Korea. Mean differences (score at the end of one-year follow up - score at baseline) in the Clinical Dementia Rating Sum of Boxes (CDRSOB) were used as the primary efficacy measures. Patients initially received $5 \mathrm{mg}$ /day donepezil for first 1 month, then $10 \mathrm{mg} /$ day for the remainder of the study.
\end{abstract}

Results: Declining rates of the CDR 0.5 and the CDR 1 have higher declining rate in CDRSOB than the CDR 2 group.

Conclusion: This study shows that the rate of cognitive decline is less in moderate $\mathrm{AD}$ than other stage of $\mathrm{AD}$. This suggests that treatment effect of donepezil may be greater in moderate stage than in mild stage of $\mathrm{AD}$.

Keywords: Alzheimer's disease; Moderate stage; Donepezil; Clinical dementia rating sum of boxes

\section{Introduction}

Alzheimer's disease (AD) is a progressive disease with an

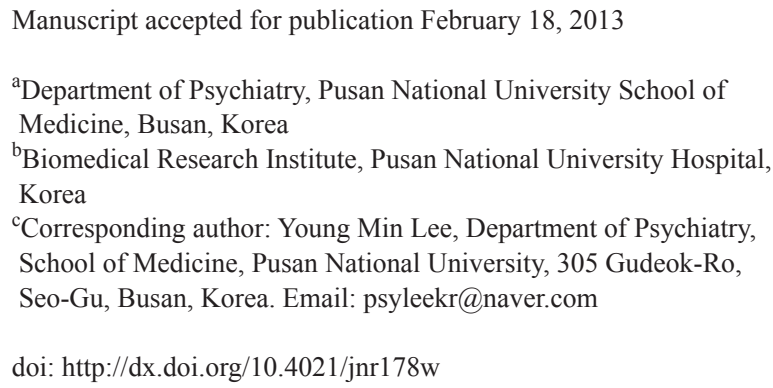

insidious onset and a downhill course. However, the previous longitudinal studies indicate that the rate of cognitive decline over the entire course of the disease is distinctly nonlinear $[1,2]$. The rate of cognitive decline was less for patients with mild stage or with severe stage than for patients with moderate stage [1].

Cholinesterase inhibitors (ChEIs), which reduce the breakdown of acetylcholine in the brain, are widely considered the treatment of choice for AD. Donepezil, galantamine, and rivastigmine are currently the three most commonly prescribed cholinesterase inhibitors. ChEIs delay cognitive and functional deterioration and decrease caregiver stress in mild to moderate stage of AD patients $[3,4]$. Donepezil, specifically, has shown significant slower decline than placebo in cognitive and functional deterioration in severe stage of $\mathrm{AD}$ patients $[5,6]$.

Many clinical studies reported that treatment response to ChEIs vary according to the severity of AD. Some studies reported that the beneficial effect of ChEIs treatment, in terms of cognition and functionality, is greater for mild than for moderate $\mathrm{AD}[7,8]$. On the other hand, studies using regression models predicted that patients with mild to moderate $\mathrm{AD}$ treated with ChEIs would show greater cognitive improvement in more advanced than in milder stages [9-11].

Treatment response seems to depend on severity degree but it is not clear which stage is more responsive. Accordingly, the aim of this study was to compare cognition and functionality outcomes after 1-year donepezil treatment among various severities of $\mathrm{AD}$ patients.

\section{Methods}

\section{Study subjects}

Subjects were recruited from the memory impairment clinic at the Department of Psychiatry, Pusan National University Hospital, Busan, Korea between April 2008 and December 2011. The inclusion criteria were: (1) age above 55 years; (2) diagnosis of $\mathrm{AD}$ according to the NINCDS-ADRDA (National Institute of Neurological and Communicative Disorders and Stroke-Alzheimer's Disease and Related Disor- 
ders Association) [12]; (3) minimal white matter ischemia (caps or band $<5 \mathrm{~mm}$ and deep white lesion $<10 \mathrm{~mm}$ ) as assessed by magnetic resonance imaging (MRI) to minimize the vascular etiologic impact; (4) no depression according to the Korean version of Mini International Neuropsychiatric Interview (MINI-K) [13, 14] at baseline examination. The exclusion criteria were: (1) subjects with clinically active cerebrovascular disease or with other conditions causally related to cognitive impairment (for example, severe organ failure, metabolic or hematologic disorders, dementia with Lewy bodies, frontotemporal dementia, Parkinson's disease, or epilepsy) at baseline examination; (2) diagnostic transitions in AD to other states or type of cognitive disorder ( for example, normal, mild cognitive impairment, vascular dementia or other dementia) during follow-up period; (3) new diagnosis for depression according to the Korean version of Mini International Neuropsychiatric Interview (MINI-K) $[13,14]$ during follow-up period. Written informed consent was obtained from all the participants, and this study was approved by the Pusan National University Hospital Institutional Review Board.

\section{Study design}

This was a 1 year, retrospective cohort study. Patients were screened within a 2 weeks of entry and were divided into three dementia severity groups by their clinical rating scales (CDR) scores at baseline: very mild (CDR 0.5), mild (CDR 1), moderate (CDR 2). Patients initially received $5 \mathrm{mg} /$ day donepezil for first $1 \mathrm{month}$, then $10 \mathrm{mg}$ /day for the remainder of the study.

\section{Baseline examination}

All patients underwent a comprehensive evaluation consisting of the following assessments: history taking from the patient and an informant; medical and neurological examinations; Korean versions of the mini-mental state examination (K-MMSE) [15, 16] for global cognitive evaluation; the Barthel-Activities of Daily Living (B-ADL) $[9,10]$ and the Seoul-Instrumental Activities of Daily Living (S-IADL) [11] for functional evaluation; the Clinical Dementia Rating (CDR) scale $[17,18]$, and the Clinical Dementia Rating Sum of Boxes (CDRSOB) [17, 18] for evaluation of dementia severity; the Consortium to Establish a Registry for Alzheimer Disease neuropsychological battery (CERAD-NP) [19, 20] or the Seoul Neuropsychological Screening Battery (SNSB) for comprehensive neuropsychological test [21]. The SNSB includes tests that assess attention, language, praxis, Gerstmann syndrome, calculation, body part identification, visuospatial function, verbal and visual memory, and executive functions. The tests that provided numerical scores included that for orientation (time and place), digit span (forward and backward) and the Korean version of the Boston Nam- ing Test, the Rey-Osterrieth Complex Figure Test (copying, immediate and 20-min delayed recall and recognition), the Seoul Verbal Learning Test (3 learning-immediate recall trials of a 12 item list, a 20-min delayed recall trial for the 12 items and recognition testing), contrasting program test/gono-go test, and a test of semantic fluency (animals and supermarket items) and letter-phonemic fluency (the Controlled Oral Word Association Test). The Stroop test (correct number of responses for word reading and naming the color of the font for 112 items during a 2-min period) was also used [21]. The K-MMSE used in this study was a version that has been previously modified to some extent for use in Korea and has been shown to exhibit properties similar to those of the original MMSE $[15,16]$. The SIADL consist of 15 items that address an individual's ability to engage in more complex tasks, such as shopping or using the telephone, and impairment severity is scored from 1 (no impairment) to 3 for all items. Thus, the maximum score of SIADL is 45 and scores of $\leq 7$ indicate normal complex ADL [22]. Additionally, laboratory tests and brain MRI were used to confirm that there were no secondary causes of cognitive impairment.

\section{Follow-up examinations}

Patients were generally evaluated after 12 months from the baseline evaluation. Follow-up examination consisted of the following assessments: history taking from the patient and from an informant; medical and neurological examinations; Korean versions of the mini-mental state examination (K-MMSE) [15, 16] for global cognitive evaluation; the Barthel-Activities of Daily Living (B-ADL) $[9,10]$ and the Seoul-Instrumental Activities of Daily Living (S-IADL) [11] for functional evaluation; the Clinical Dementia Rating (CDR) scale [17, 18], and the Clinical Dementia Rating Sum of Boxes (CDRSOB) [17, 18] for evaluation of dementia severity; the Consortium to Establish a Registry for Alzheimer Disease neuropsychological battery (CERAD-NP) [19, 20] or the Seoul Neuropsychological Screening Battery (SNSB) for comprehensive neuropsychological test [21].

The major goal of the follow-up examination was to evaluate deterioration rate due to neurodegenerative etiology in AD. Therefore, this study exclude other causes of deterioration except neurodegenerative etiology (for example, head trauma, stroke, intracranial hemorrhage, depression, drug or other metabolic diseases) in order to focus on the deterioration of $\mathrm{AD}$ due to neurodegenerative etiology during the follow-up period

\section{Data analysis}

Demographic and clinical variables were compared using Chi-square tests for categorical variables, and the KruskalWallis test for variables that were not normally distributed; multiple comparisons were performed using the least sig- 
Table 1. Baseline Characteristics of the Patients With Alzheimer's Disease, Mean \pm S.D. or (\%)

\begin{tabular}{lllll}
\hline \multirow{2}{*}{ Varibles } & \multicolumn{3}{c}{ Severity of Alzheimer's disease } \\
\cline { 2 - 5 } & Very mild(CDR 0.5) & Mild(CDR 1) & Moderate(CDR 2) \\
\hline Number & 188 & 153 & 31 & \\
Age, years & $72.02 \pm 8.15$ & $72.54 \pm 8.04$ & $77.06 \pm 6.60$ & 0.007 \\
Male gender, \% & 37.0 & 26.3 & 19.4 & 0.033 \\
Education, years & $8.45 \pm 5.54$ & $6.80 \pm 5.14$ & $5.81 \pm 4.83$ & 0.003 \\
Baseline K-MMSE score & $21.95 \pm 3.63$ & $17.65 \pm 4.55$ & $13.61 \pm 3.74$ & $<0.001$ \\
Baseline CDR-SOB & $3.07 \pm 0.88$ & $5.92 \pm 1.25$ & $10.63 \pm 1.62$ & $<0.001$ \\
Baseline IADL & $11.97 \pm 5.56$ & $21.14 \pm 8.48$ & $30.16 \pm 9.84$ & $<0.001$ \\
Baseline basic ADL & $19.88 \pm 0.54$ & $19.10 \pm 2.27$ & $17.81 \pm 3.14$ & $<0.001$ \\
Average period of follow-up, years & $1.02 \pm 0.08$ & $1.01 \pm 0.08$ & $1.02 \pm 0.10$ & 0.644
\end{tabular}

nificant difference test with ranks. We applied analysis of covariance (ANCOVA) with covariates of the ages, gender and education and the least significant difference test for post hoc comparisons to identify between-three dementia severity groups (CDR 0.5, CDR 1 and CDR 2) in cognition and function (K-MMSE, B-ADL, S-IADL, CDRSOB) between baseline and the end of one-year follow up. All analysis was performed using SPSS for Windows, version 15.0 (SPSS Inc., Chicago, IL). Statistical tests were 2-tailed, and results were regarded as significant at or below the $5 \%$ probability level $(\mathrm{P}<0.05)$

\section{Results}

Table 1 shows the demographic and clinical characteristics of the patients with AD. A total of 188 subjects (51\%) had very mild AD at baseline (CDR 0.5$), 153$ subjects ( $41 \%$ ) had mild AD (CDR 1) and 31 subjects (8\%) had moderate AD (CDR 2). Significant difference in mean age, gender, education, and clinical variables (K-MMSE, B-ADL, S-IADL, CDRSOB) was found between the 3 severity groups of AD (CDR 0.5, CDR 1 and CDR 2). Subjects with a CDR 2 were 5 years older than those with a CDR 0.5 (74.55 \pm 7.39 vs. $\left.72.02 \pm 8.15 ; \chi^{2}=11.53 ; \mathrm{P}=0.007\right)$. The frequency of male gender was less by $17.6 \%$ in subjects with a CDR 2 than in those with a CDR $0.5\left(37 \%\right.$ vs. $\left.19.4 \% ; \chi^{2}=6.79 ; \mathrm{P}=0.033\right)$. The level of education was less by 2.6 years in subjects with a CDR 2 than in those with a CDR 0.5 (5.81 \pm 4.83 vs. 8.45 \pm 5.54; $\chi^{2}=9.79 ; \mathrm{P}=0.003$ ).

Table 2 shows mean differences (score at the end of one-year follow up - score at baseline) in K-MMSE score, CDRSOB score, S-IADL score and B-ADL scores for each of the three dementia severity groups by their CDR scores. Declining rates of the K-MMSE score seem higher in the CDR 0.5 and the CDR 1 groups than in the CDR 2 group, and Declining rates of the S-IADL score seem higher in the CDR 0.5 than in the CDR 1 groups and the CDR 2 group, even though these between-group differences are not statistically significant. The CDR 2 group has a higher declining rate in $\mathrm{B}-\mathrm{ADL}$ than the CDR 0.5 and the $\mathrm{CDR} 1$ groups $(\mathrm{F}=$ 2.94, $\mathrm{P}=0.01)$, but the CDR 0.5 and the CDR 1 have higher declining rate in CDRSOB than the CDR 2 group $(\mathrm{F}=2.77$, $\mathrm{P}=0.04)$

\section{Discussion}

Treatment response to ChEIs seems to depend on severity degree of $\mathrm{AD}$ but it is not clear which stage is more responsive. To the best of our knowledge, this is the first study to compare cognition and functionality outcomes after 1-year donepezil treatment among various severities of AD patients in Korea.

In this study, the declining rate in cognition, as measured by the K-MMSE, is not consistent throughout the disease process, showing higher mean difference in the very mild (1.37 point per year) and to mild groups (1.27 point per year) than in the moderate group ( 0.42 point per year), even though this between-group difference was not statistically significant (Table 2). In the CDR-SOB, the lowest mean differences were also seen in the moderate group, and this between-group difference was statistically significant $(\mathrm{P}=$ 0.04). Those mean that the greatest effects of donepezil are seen in the moderate group of AD.

These findings are not consistent with previous stud- 


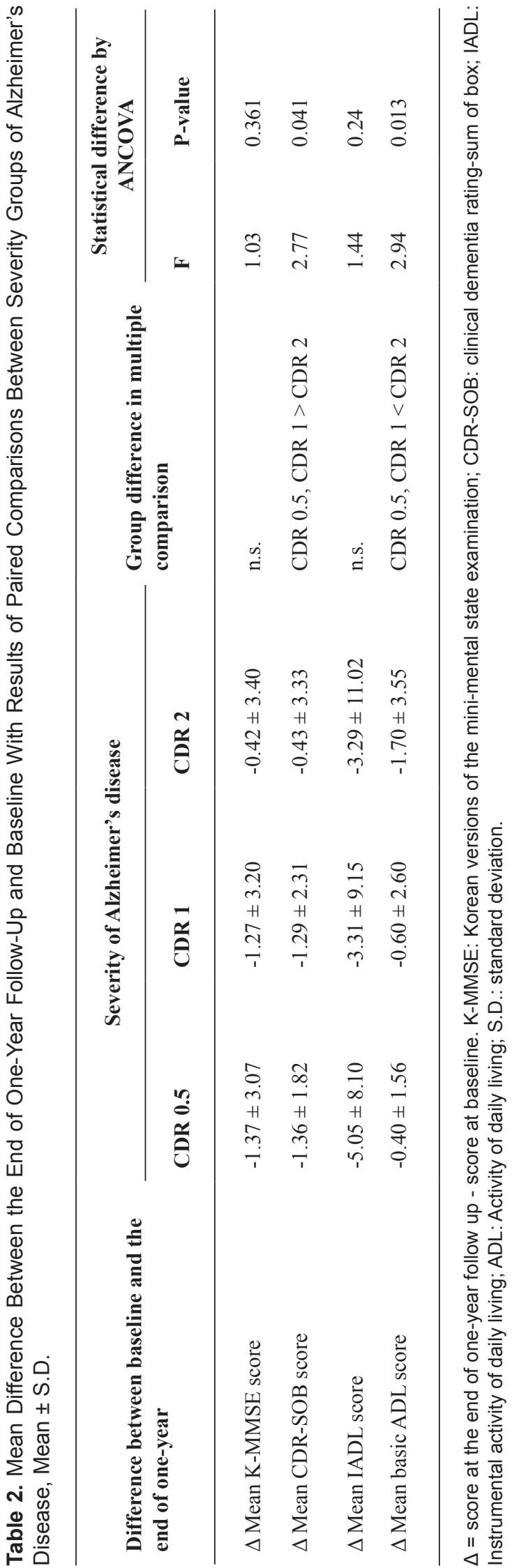

ies carried out in a number of other countries and settings $[7,8]$. In a 6-month prospective, observational, multicenter study [7], Molinuevo and his colleagues reported that the beneficial effect of donepezil treatment, in terms of cognition and functionality, is greater for mild than for moderate AD. They showed a greater benefit of donepezil treatment in the mild over the moderate $\mathrm{AD}$ group with significant differences between the worsening (decreased scores) observed in Temporal orientation and Semantic memory domains in the moderate $\mathrm{AD}$ group and the improvement (increased scores) in the mild $\mathrm{AD}$ group.

However, their study is short period and no placebo controlled. Due to the short period of study, their study is should be considered preliminary and required replication with longer follow-up. In addition, due to open label no placebo controlled study, it is difficult to establish the causal relationship between severity of disease and treatment response. In the natural course of $\mathrm{AD}$, the rate of cognitive decline was less for patients with mild stage or with severe stage than for patients with moderate stage [1]. Since, they compared the rate of cognitive decline after 6-month donepezil treatment in mild versus moderate AD patients without placebo groups, interpretation of the findings requires caution.

In the multicenter, double-blind, placebo-controlled, 26week studies [11], Kurz and his colleagues reported that the greatest rivastigmine versus placebo differences with regard to cognitive performance scores were seen in the moderately severe cohort. These findings are consistent with those of our study that the greatest effects of donepezil are seen in the moderate group of $\mathrm{AD}$. Cholinergic deficiency is the most severe and consistent biochemical change in Alzheimer's disease. This is seen as reduced levels of acetylcholine, choline acetyltransferase, and acetylcholinesterase, reported in both necropsy brain samples and in cerebrospinal fluid [23, 24]. However, in the mild cognitive impairment and the early $\mathrm{AD}$, the mean acetylcholinesterase activity was higher than in the moderate $\mathrm{AD}[25,26]$. It may be that in mild $\mathrm{AD}$ and mild cognitive impairment there is relative upregulation or compensation of acetylcholinesterase. That means that cholinergic deficits were not apparent in patients with mild AD. Therefore, treatment effect of donepezil may be greater in moderate stage than in mild stage of AD.

The present study has several limitations. First, our study was conducted on subjects recruited from specialized memory disorder clinics, and therefore, we may not be able to generalize them for the whole population of $\mathrm{AD}$ patients. Second, in our study, the percentage of moderate AD from total $\mathrm{AD}$ was $8.3 \%$ and is somewhat lower than that of other stage AD. Third, the follow up period was relatively short. Fourth, there could have been learning effects in this study, since the neuropsychological workup included the same battery at baseline and follow-up. Additionally, the APOE genotype and other factors which influence cognitive decline in $\mathrm{AD}$ were not included as covariates in our analysis. Finally, 
since this study is observational cohort study, interpretation of our findings requires caution.

In conclusion, in the natural course of $\mathrm{AD}$, the rate of cognitive decline was highest for patients with moderate stage. However, after 1-year donepezil treatment, our study showed that the rate of cognitive decline was less in moderate $\mathrm{AD}$ than other stage of AD. This suggested that treatment effect of donepezil may be greater in moderate stage than in mild stage of AD.

\section{Acknowledgement}

This study was supported by Biomedical Research Institute (2009-27), Pusan National University Hospital.

\section{References}

1. Stern RG, Mohs RC, Davidson M, Schmeidler J, Silverman J, Kramer-Ginsberg E, Searcey T, et al. A longitudinal study of Alzheimer's disease: measurement, rate, and predictors of cognitive deterioration. Am J Psychiatry. 1994;151(3):390-396.

2. Morris JC, Edland S, Clark C, Galasko D, Koss E, Mohs $\mathrm{R}$, van Belle $\mathrm{G}$, et al. The consortium to establish a registry for Alzheimer's disease (CERAD). Part IV. Rates of cognitive change in the longitudinal assessment of probable Alzheimer's disease. Neurology. 1993;43(12):24572465.

3. Birks J. Cholinesterase inhibitors for Alzheimer's disease. Cochrane Database Syst Rev. 2006;(1):CD005593.

4. Hansen RA, Gartlehner G, Lohr KN, Kaufer DI. Functional outcomes of drug treatment in Alzheimer's disease: A systematic review and meta-analysis. Drugs Aging. 2007;24(2):155-167.

5. Feldman H, Gauthier S, Hecker J, Vellas B, Emir B, Mastey V, Subbiah P. Efficacy of donepezil on maintenance of activities of daily living in patients with moderate to severe Alzheimer's disease and the effect on caregiver burden. J Am Geriatr Soc. 2003;51(6):737-744.

6. Tariot PN, Cummings JL, Katz IR, Mintzer J, Perdomo CA, Schwam EM, Whalen E. A randomized, double-blind, placebo-controlled study of the efficacy and safety of donepezil in patients with Alzheimer's disease in the nursing home setting. J Am Geriatr Soc. 2001;49(12):1590-1599.

7. Molinuevo JL, Berthier ML, Rami L. Donepezil provides greater benefits in mild compared to moderate Alzheimer's disease: implications for early diagnosis and treatment. Arch Gerontol Geriatr. 2011;52(1):18-22.

8. Saumier D, Murtha S, Bergman H, Phillips N, Whitehead V, Chertkow H. Cognitive predictors of donepezil therapy response in Alzheimer disease. Dement Geriatr
Cogn Disord. 2007;24(1):28-35.

9. Wattmo C, Hansson O, Wallin AK, Londos E, Minthon L. Predicting long-term cognitive outcome with new regression models in donepezil-treated Alzheimer patients in a naturalistic setting. Dement Geriatr Cogn Disord. 2008;26(3):203-211.

10. Feldman HH, Jacova C. Predicting response to acetylcholinesterase inhibitor treatment in Alzheimer disease: has the time come? Nat Clin Pract Neurol. 2009;5(3):128-129.

11. Kurz A, Farlow M, Quarg P, Spiegel R. Disease stage in Alzheimer disease and treatment effects of rivastigmine. Alzheimer Dis Assoc Disord. 2004;18(3):123-128.

12. McKhann G, Drachman D, Folstein M, Katzman R, Price D, Stadlan EM. Clinical diagnosis of Alzheimer's disease: report of the NINCDS-ADRDA Work Group under the auspices of Department of Health and Human Services Task Force on Alzheimer's Disease. Neurology. 1984;34(7):939-944.

13. Sheehan DV, Lecrubier Y, Sheehan KH, Amorim P, Janavs J, Weiller E, Hergueta T, et al. The Mini-International Neuropsychiatric Interview (M.I.N.I.): the development and validation of a structured diagnostic psychiatric interview for DSM-IV and ICD-10. J Clin Psychiatry. 1998;59(Suppl 20):22-33; quiz 34-57.

14. Choi YH, Yoo, S.W., Kim, Y.S. Validity of Korean Version of the Mini-International Neuropsychiatric Interview. Anxiety and Mood 2006; 2: 50-55.

15. Folstein MF, Folstein SE, McHugh PR. "Mini-mental state". A practical method for grading the cognitive state of patients for the clinician. J Psychiatr Res. 1975;12(3):189-198.

16. Han C, Jo SA, Jo I, Kim E, Park MH, Kang Y. An adaptation of the Korean mini-mental state examination (KMMSE) in elderly Koreans: demographic influence and population-based norms (the AGE study). Arch Gerontol Geriatr. 2008;47(3):302-310.

17. Morris JC. The Clinical Dementia Rating (CDR): current version and scoring rules. Neurology. 1993;43(11):24122414.

18. Choi SH ND, Lee BH, Hahm DS, Jeong JH, Yoon SJ, Yoo KH, Ha CK, Han IW, Dementia Research Group. Estimating the Validity of the Korean Version of Expanded Clinical Dementia Rating (CDR) Scale. J Korean Neurol Assoc 2001; 19: 585-591.

19. Lee JH, Lee KU, Lee DY, Kim KW, Jhoo JH, Kim JH, Lee $\mathrm{KH}$, et al. Development of the Korean version of the Consortium to Establish a Registry for Alzheimer's Disease Assessment Packet (CERAD-K): clinical and neuropsychological assessment batteries. J Gerontol B Psychol Sci Soc Sci. 2002;57(1):P47-53.

20. Lee DY, Lee KU, Lee JH, Kim KW, Jhoo JH, Kim SY, Yoon JC, et al. A normative study of the CERAD neuropsychological assessment battery in the Korean elderly. 
J Int Neuropsychol Soc. 2004;10(1):72-81.

21. Ahn HJ, Chin J, Park A, Lee BH, Suh MK, Seo SW, Na DL. Seoul Neuropsychological Screening Batterydementia version (SNSB-D): a useful tool for assessing and monitoring cognitive impairments in dementia patients. J Korean Med Sci. 2010;25(7):1071-1076.

22. Ku HM KJ, Kwon EJ, Kim SH, Lee HS, Ko HJ, Jo SM, Kim TK. A Study on the Reliability and Validity of SeoulInstrumental Activities of Daily Living (SIADL). Neuropsychiatr Assoc 2004; 43: 189-199.

23. Perry EK, Perry RH, Blessed G, Tomlinson BE. Necropsy evidence of central cholinergic deficits in senile dementia. Lancet. 1977;1(8004):189.
24. Francis PT, Palmer AM, Snape M, Wilcock GK. The cholinergic hypothesis of Alzheimer's disease: a review of progress. J Neurol Neurosurg Psychiatry. 1999;66(2):137-147.

25. Rinne JO, Kaasinen V, Jarvenpaa T, Nagren K, Roivainen A, Yu M, Oikonen V, et al. Brain acetylcholinesterase activity in mild cognitive impairment and early Alzheimer's disease. J Neurol Neurosurg Psychiatry. 2003;74(1):113-115.

26. Davis KL, Mohs RC, Marin D, Purohit DP, Perl DP, Lantz M, Austin G, et al. Cholinergic markers in elderly patients with early signs of Alzheimer disease. JAMA. 1999;281(15):1401-1406. 